\title{
Criterios de selección para recursos digitales
}

\author{
María del Carmen Negrete Gutiérrez \\ Centro Universitario de Investigaciones Bibliotecológicas \\ Universidad Nacional Autónoma de México
}

\subsection{Resumen}

En el actual ambiente tecnológico, varios de los factores prevalecientes para la toma de decisiones en la formación de colecciones en las bibliotecas universitarias están cambiando. En este contexto, se abordan diversos fenómenos involucrados con el proceso de selección ocasionados por el advenimiento de los recursos digitales. Se reflexiona sobre el papel que deben jugar los responsables de tomar decisiones relacionadas con materiales impresos y recursos digitales en la biblioteca universitaria. Finalmente, se presentan una serie de criterios para evaluar y seleccionar recursos basados en Web. (Autor)

Palabras clave: Bibliotecas universitarias. Selección. Recursos digitales.

\subsection{Abstract}

In the current technological environment, several factors affecting decision taking in collection development for university libraries are changing quickly. Such factors involving digital resources and related to the selection processes are analysed. The role of those responsible for deciding about printed and digital resources acquisition is considered. Finally, a criteria set for evaluating and selecting web-based resources is presented. (Author)

Keywords: University libraries. Seleccion. Digital resources.

\section{Introducción}

El objetivo de las bibliotecas académicas es apoyar la enseñanza, el aprendizaje, la academia, la investigación y cubrir otras necesidades de información de sus profesores, estudiantes, investigadores y asociados, de manera efectiva y eficiente. En la actualidad, las bibliotecas académicas, principalmente, se enfrentan al crecimiento de la oferta de la información con costos crecientes que afectan, en ocasiones, a los limitados recursos presupuestales con que cuentan para realizar sus adquisiciones. A esto hay que aunar el propio auge de los diversos medios electrónico/digitales que, si bien permiten una mayor cobertura, diseminación, 
acceso y transferencia de la información, también representan, en buena medida, costos mayores para su adquisición o suscripción. Los recursos electrónico/digitales, indudablemente, están impactando no solamente a la organización de la biblioteca, la estructura de sus departamentos, sus funciones, procesos y el modo en que se ofrecen los servicios a los usuarios, sino también al sistema de comunicación tradicional.

En el actual ambiente tecnológico, varios de los factores prevalecientes para la toma de decisiones en la formación de colecciones están cambiando, lo que hace necesario que el documentalista y el bibliotecólogo desarrollen nuevas habilidades que le permitan utilizar la tecnología y tener conocimiento permanente de las fuentes especializadas que se están generando y que les pueden apoyar en el proceso de selección. Todo ello permitirá detectar no solamente la información que se requiere, sino también el formato o medio idóneo en la que está disponible. Los bibliotecólogos y documentalistas están ampliando el rango de recursos que pueden ofrecerle a su comunidad, al hacer disponibles recursos en formato electrónico/digital, los cuales tienen una clara ventaja sobre los materiales impresos, debido a la facilidad que otorgan para la localización y manipulación de la información. Sin embargo, obtener información electrónico/digital para satisfacer las demandas de información requiere que la biblioteca cuente con presupuesto suficiente, con infraestructura adecuada de equipo, programas, apoyo profesional y licencias de acceso a diversos servicios y productos que se ofrecen actualmente. Ante estas condicionantes, la selección de recursos digitales —especialmente de los recursos de información accesibles a través de una sede Web- se vuelve más complicada por las diversas características que reúnen este tipo de recursos, además de las necesidades específicas de herramientas o equipos de acceso.

En resumen, puede decirse que las bibliotecas académicas necesitan buscar nuevas formas y técnicas para optimizar sus presupuestos ante los servicios y productos disponibles en el mercado informativo. Se reconoce que las nuevas tecnologías de información presentan tanto oportunidades como retos: oportunidades por la cantidad de información potencial que se puede manipular; y retos en términos de la capacidad para seleccionarla, además de la inversión significativa que se requiere, tanto económica como de factor humano, particularmente en capacitación. La transición de la disponibilidad de la publicación impresa a la distribución digital de recursos académicos y de investigación, implica cuestionamientos que no se pueden ignorar o dejar de lado. Por tanto, es necesario describir los elementos fundamentales de la información digital con el propósito de que su contenido responda a las necesidades y demandas informativas de los usuarios finales, ya sean éstos estudiantes, profesores, investigadores, documentalistas o bibliotecólogos. El presente trabajo se enfoca a la selección de recursos existentes en una sede Web, independientemente de que sean gratuitos o no.

Scire. $8: 2$ (jul.-dic. 2002) 53-60. 


\section{El proceso de selección}

La práctica de la selección de recursos es una de las funciones mas antiguas y fundamentales del desarrollo de colecciones, ya que a través de ella se ha garantizado el control de calidad de los materiales que han ingresado a la biblioteca. El concepto de selección se define como el proceso que permite evaluar la calidad, importancia y/o utilidad del contenido de los recursos, con el objeto de que éstos respondan de forma adecuada a las necesidades y demandas de información que tiene la comunidad a la cual la biblioteca debe atender (Negrete, 1999, p. 57).

En la actualidad, la selección de recursos exige un mayor compromiso profesional por parte de los responsables en la toma de decisiones debido, entro otros aspectos, "[...] al crecimiento exponencial de la información en línea, a la existencia de una tipología documental múltiple, a la inestabilidad debida a continuas actualizaciones que conlleva su disponibilidad temporal, y al requerimiento de confiabilidad" (Garduño, 2001). Con el advenimiento de los recursos de información digital, las prácticas de la selección se han ampliado al introducir nuevos cambios. Ahora se tiene que escoger entre formatos, identificar que está disponible, analizar costos, tener un entendimiento de licencias, derechos de autor y otros aspectos legales, interpretar las implicaciones del servicio, y preparar el equipo y las facilidades (Jenkins y Morley, 1999, p. 115). Ahora, más que nunca, el proceso de selección requiere de criterios apropiados, claros y consistentes que ayuden a los responsables a tomar de decisiones adecuadas para ingresar materiales a la biblioteca, pero también para tomar decisiones sobre recursos digitales que permitan el acceso, recuperación y uso de la información digital.

El proceso de selección no parte de una metodología específica que pueda generalizarse a todo tipo de biblioteca o a las de un mismo tipo, ya que depende de los objetivos de cada una de ellas y de su organización interna; de las tendencias de desarrollo que emita la institución de la que depende; de la tasa de crecimiento de su comunidad; del presupuesto asignado, de su infraestructura tecnológica y del elemento humano que hace operable este proceso. El crecimiento de la información y su representación en una diversidad de formatos, influye en las formas tradicionales de llevar a cabo la selección de recursos. Esta se complica por la dificultad de normalizar este proceso, dado que involucra una serie de decisiones que podrían no ser válidas para todo tipo de recursos. Por ello, se hace indispensable contar con criterios de selección claramente definidos que permitan dar juicios de valor que sean lo más objetivos y coherentes posible. Se debe buscar formar colecciones equilibradas con los mejores recursos impresos y con los mejores recursos digitales, lo que requerirá un trabajo responsable y efectivo para responder a los intereses de los usuarios de la biblioteca. Los responsables de la selección deben tomar en cuenta aspectos como los siguientes:

Scire. $8: 2$ (jul.-dic. 2002) 53-60. 
- Establecer claramente la misión y objetivos de la biblioteca.

- Identificar las necesidades reales y potenciales de su comunidad.

- Identificar las fortalezas y debilidades de la colección, en términos de áreas o materias y de recursos específicos.

- Determinar qué presupuesto está disponible para el desarrollo de colecciones impresas y digitales.

- Determinar con qué presupuesto se cuenta para infraestructura tecnológica.

- Desarrollar un plan para identificar el uso potencial de los diferentes recursos que podrían adquirirse o a los que podría suscribirse.

- Realizar un estudio sobre los servicios que se usan: ¿cómo se usan? y ¿cuáles se desean?

Aunado a lo anterior, requieren mantenerse actualizados sobre el comportamiento editorial nacional y extranjero, y sobre la forma en que operan sus diferentes procesos de producción, diseminación, tiempos y costos; conocer de los editores su autoridad y seriedad, las áreas y niveles en las que producen, políticas de inclusión, tipos de propaganda que emplean, entre otros; así como un conocimiento de los distribuidores, productores, vendedores y proveedores, y de los servicios y productos que ofrecen.

Es indudable que la experiencia personal de los selectores a través del tiempo, el conocimiento de la información que se genera en las áreas o temas de interés y la aplicación de criterios, les ha permitido determinar o asignar un valor intrínseco a los recursos. Es evidente que cuando el selector se enfrenta a la información representada en formato digital, tendrá que aplicar los criterios tradicionales complementados con otros adicionales y dar juicios objetivos de acuerdo a los elementos básicos que deben evaluarse para cada uno de los recursos informativos. Sin duda, la selección de recursos digitales no es responsabilidad exclusiva del bibliotecólogo o documentalista, ni tampoco del profesor/investigador. La responsabilidad debe ser compartida entre todos, particularmente por quienes están en el servicio de consulta o referencia, en el servicio de publicaciones periódicas o en el servicio de diseminación selectiva de información, por el solo hecho de estar en contacto directo con los usuarios y sus necesidades y/o demandas de información de manera permanente. La utilización de tecnologías de información en los servicios que ofrecen las bibliotecas obliga a los selectores de materiales a conocer las nuevas tendencias del desarrollo de colecciones debido, sobre todo, a que ahora la demanda de información de su comunidad es también de recursos digitales. Lluís Codina (2000, p. 12) señala que, en la "medida que la Internet vaya creciendo, será más y más necesario para los profesionales de la Documentación (y de la Bibliotecología) disponer de criterios sólidos para saber evaluar recursos digitales 
y determinar su valor o su capacidad relativa para cumplir sus objetivos". Actualmente, seleccionar un recurso digital no es tan directo como seleccionar un recurso impreso, ya que involucra un amplio número de aspectos adicionales, además de la apropiada información contenida en el recurso y de la ayuda que ofrece cada uno. Ahora se requiere un equipo de profesionales capacitados en el uso de la tecnología, de la información, de la didáctica, de la lectura visual y de las herramientas propias de la profesión y, por supuesto, con una fuerte vinculación al trabajo académico dentro y fuera de la biblioteca, lo que permitirá a su vez, desarrollar habilidades, actitudes, valores y conocimientos en sus usuarios, que les permita llevar a cabo el uso, acceso y transformación de la información en un conocimiento útil para la toma de decisiones y para resolver problemáticas diversas.

De acuerdo con Pedro Hípola, el término recurso digital incluye: “[...] texto, datos numéricos, ilustraciones, fotografías, sonido, video, diapositivas [...], en definitiva, cualquier ejemplo de "objeto digital". Estos objetos representan la información almacenada en los depósitos documentales y están compuestos por su propio contenido (bits), metadatos o propiedades asociadas a ellos, un registro de operaciones realizadas y etiquetas para su identificación" (Hípola, VargasQuesada y Senso, 2000, p. 7). La tipología de recursos digitales es muy variada, y es considerada desde puntos de vista muy distintos. Una posible agrupación de recursos digitales en Internet es la proporcionada por Lluís Codina (2000, p. 15), quién reconoce que está inacabada, y propone los siguientes: bases de datos, directorio, documento, medio de comunicación, motor de búsqueda, organización no gubernamental (ONG), publicación periódica, sede web corporación, sede web institución académica y sede web institución comercial. Generalmente, las colecciones de recursos digitales aparecen como un sitio Web o como una compilación de ligas de otros servidores. Por ejemplo, una colección basada en el Web incluiría, entre otras, ligas a:

1. Recursos impresos como directorios y bibliografías, incluyendo recursos de consulta o colecciones especiales que se han convertido a páginas Web o en otro formato electrónico.

2. Un conjunto de servicios, que han sido adaptados a un ambiente en línea: referencia, préstamo interbibliotecario y obtención de documentos, etc.

3. Recursos internos que han sido digitalizados: listas de reserva, información descriptiva sobre la biblioteca y su acervo, literatura gris, etc.

4. Contenido específicamente desarrollado para el ambiente Web, tales como tutoriales interactivos, documentos hipertextuales, libros de texto en línea, talleres o cursos. 
5. Colecciones de valor añadido con ligas a recursos Web que están organizados de una manera orientada a los usuarios de una biblioteca.

6. Recursos electrónicos que se obtienen a través del Web, que pueden ser gratuitos o no, tales como: bases de datos bibliográficas, de texto completo o de imágenes (Kovacs y Elkordy, 2000, p. 336).

Conforme se populariza el recurso digital a través del Web, se necesita conjuntar bibliotecólogos, documentalistas, profesionales de la información, especialistas en cómputo e ingenieros, no solo para realizar una adecuada selección de recursos, sino también para seleccionar otras herramientas que faciliten la descripción y recuperación de esos recursos digitales.

Ahora, debemos ajustar la mayor parte de nuestras prácticas profesionales y operaciones diarias para manejar los recursos electrónico/digitales con una facilidad igual o mayor que como lo hemos realizado con recursos impresos.

\section{Criterios de selección}

Tradicionalmente, los criterios que se han aplicado para seleccionar materiales impresos son, entre otros: autoridad del autor, importancia del tema, cobertura, actualidad de la información, nivel de tratamiento, estilo, formato y costo. Ahora nos preguntamos ¿éstos criterios siguen prevaleciendo o habría que cambiarlos o complementarlos? La revisión de los trabajos de Codina, Kovacs, White y Crawford permitió resumir criterios para evaluar y seleccionar recursos basados en Web. Bajo cada criterio se señalan una serie de preguntas o cuestionamientos que el evaluador deberá responder (Codina, 2000, p. 29-3; Kovacs, 2000, p. 345-347; White y Crawford, 1997, p. 53-57).

Contenido: ¿Posee un valor o interés intrínseco? La información que se presenta en el sitio ¿está tratada con el cuidado y rigor adecuados al contenido? La información ¿es útil en el formato que se presenta? ¿La información está en un lenguaje apropiado y con una profundidad accesible para la población a la que se dirige? ¿Posee un adecuado grado de cobertura de la información, de acuerdo al tema tratado? ¿Las fuentes citadas están documentadas?

Autoria: ¿El recurso dispone de título y fecha de publicación? ¿Contiene indicaciones claras y explícitas sobre la autoría y/o responsabilidad intelectual del recurso? ¿Hay una autoridad claramente identificada como responsable de su mantenimiento? ¿Existen indicios de que el recurso está creado, producido o editado por una institución u organismo de reconocido prestigio en su campo? ¿Se incluye texto explicativo del contenido de la Web?

Actualidad: ¿La actualización es una prioridad importante? ¿Se actualiza la información regularmente? ¿Se muestra claramente la fecha en que la fuente fué 
actualizada por última vez? ¿Se anotan las fechas de las revisiones de las ligas externas?

Facilidad de uso: ¿El sitio está accesible para usuarios con necesidades especiales? ¿Es claro cómo navegar a través de las páginas del recurso? ¿El sitio está bien organizado a lo largo de líneas lógicas? ¿Tiene un mapa de sitio? ¿Estan los botones o ítems de menús claramente etiquetados y de manera útil? Si el sitio usa "frames", ¿están aplicados de manera significativa que ayude a la navegación? ¿Hay un alto porcentaje de ligas muertas o inactivas interna o externamente? ¿La recuperación de información se hace a través de búsqueda por palabras o frases? ¿Es posible utilizar operadores booleanos? ¿Es posible utilizar otros operadores? ¿Existen ayudas y sistemas de recuperación de información adicionales? ¿Ha sido rápida la descarga de la página? ¿Qué hace excesivo el tiempo de descarga?

Presentación: ¿La información está presentada clara y lógicamente en un formato útil para la audiencia a la que va dirigida? ¿Posee el recurso un sumario general que representa su contenido global? ¿La interfaz es clara y fácil de usar? ¿Los elementos navegacionales son claramente identificados?, ¿Las ligas a otras páginas son fáciles de encontrar? ¿Cómo aparece el sitio en el "browser” (s)? ¿Esta disponible la versión de "sólo texto" en el sitio? ¿Sería difícil para los usuarios con incapacidades o necesidades especiales usar el sitio? (problemas potenciales: el tipo de letra pequeño, poco uso de espacio en blanco, frames, falta de color de contraste entre elementos, etc. ) ¿Contiene el recurso algún tipo de índice temático, cronológico, alfabético, de nombres, etc.? ¿Se usan siempre los mismos códigos textuales e icónicos para representar las mismas funciones, acciones o datos?

Interactividad: ¿Se puedan plantear preguntas, sugerencias o enviar mensajes al administrador del sitio? ¿Se puede realizar alguna clase de actividad o transacciones? ¿Permite descargar software o ficheros gráficos? ¿Contiene enlaces externos la Web? ¿Los enlaces han sido evaluados?

Apropiado: ¿Se aprovecha el recurso por las características únicas del medio? ¿Hay alguna otra obra impresa o no impresa que presente la información de mejor manera que la que tiene en este sitio? ¿El sitio contiene información digital que pueda ser usada en otras aplicaciones o formatos? (por ejemplo: gráficas, estadísticas, texto, software, presentaciones, etc. ) ¿El recurso está disponible en otros formatos, por ejemplo, una contraparte impresa para revistas? ¿Cuáles son las capacidades de búsqueda para la información en el sitio? (texto completo indizado, archivos PDF que no son recuperables) ¿Están disponibles gráficas y texto completo para los artículos? ¿El contenido necesita ser actualizado frecuentemente? ¿Qué hace a este recurso útil en formato electrónico? ¿Por qué no usarlo en formato impreso? ¿Hay alguna oferta para adquirir ambos? ¿Es una opción realista recomendarlo en este formato? ¿La comunidad usuaria pre-

Scire. $8: 2$ (jul.-dic. 2002) 53-60. 
fiere usar una versión electrónica o impresa? ¿Cuántos usuarios potenciales existen para este recurso?

Aunado a los criterios apuntados, habría que considerar dos aspectos importantes: equipo, presupuesto y espacio, es decir: ¿La biblioteca cuenta con el equipo apropiado para utilizar la versión electrónica/digital? ¿La biblioteca tiene el presupuesto para adquirir el equipo necesario? ¿Existe el espacio suficiente para todo el equipo necesario y para todos los usuarios que van a consultar ésos recursos?

Finalmente, habría que apuntar que los principios del desarrollo de colecciones impresas, no cambian radicalmente con las nuevas tecnologías de publicación, pero los métodos, los criterios y las prácticas en la selección deben ser ajustadas para incorporar recursos electrónico/digitales en los servicios que se ofrecen o se ofrecerán en las bibliotecas universitarias o especializadas.

\section{Referencias y bibliografía consultada}

Codina, Lluís (2000). Evaluación de recursos digitales en línea: conceptos, indicadores y métodos. // Revista Española de Documentación Científica. 23:1 (2000).

Davis, Trisha L. (1997). The evolution of selection activities for electronic resources. // Library Trends. 45:3 (1997) 391-403.

Garduño Vera, Roberto. (et. al) (2001). Módulo 111: Representación de la información digital: Guía de aprendizaje [Documento mecanografiado]. Ciudad Universitaria, 2001.

Hastings, S. K. (1998). Selection and evaluation of networked information resources. // Acquisitions Librarian. 20 (1998) 109-130.

Herring, Mark Y. (2001). 10 reasons why the Internet is no substitute for a library. // American Libraries (april 2001) 76-78.

Hípola, Pedro; Vargas-Quesada, Benjamín; Senso, José A. (2000). Bibliotecas digitales: situación actual y problemas. // El Profesional de la Información. 9:4 (abril 2000).

Jenkins, Clare; Morley, Mary (1999). Collection Management in Academic Libraries. 2nd ed. Great Britain: Gower, 1999.

Johnson, Peggy; Bonnie MacEvan (1994). Collection Management and Development : issues in an electronic era. Chicago: ALA, 1994. 148 p. (ALCTS Papers on Library Technical Services and Collectiones: 5).

Kovacs, Diane K.; Elkordy; Angela. Collection development in cyberspace: building an electronic library collection. // Library Hi Tech. 18:4 (2000).

Negrete Gutiérrez, María del Carmen (1999). El impacto del cambio en el desarrollo de colecciones en bibliotecas universitarias. // Scire. 5:1 (enero-junio 1999).

Nisonger, Thomas E. (1997). Electronic journal collection management issues. // Collection Building. 26: 2 (1997) 58-65.

White, Gary W.; Crawford, Gregory A. (1997). Developing an electronic information resources collection development policy. // Collection Building. 26:2 (1997) 53-37. 